\title{
Expected Transmission Energy Route Metric for Wireless Mesh Senor Networks
}

\author{
YanLiang Jin, ${ }^{1}$ HuiJun Miao, ${ }^{1}$ Quan Ge, ${ }^{1}$ and Chi Zhou ${ }^{2}$ \\ ${ }^{1}$ Key Laboratory of Special Fiber Optics and Optical Access Networks of Ministry of Education, Shanghai University, \\ Shanghai 200072, China \\ 2 Illinois Institute of Technology, Chicago, IL 60616-3793, USA \\ Correspondence should be addressed to YanLiang Jin, jinyanliang@staff.shu.edu.cn
}

Received 1 November 2010; Accepted 5 April 2011

Academic Editor: Robert C. Qiu

Copyright (๑) 2011 YanLiang Jin et al. This is an open access article distributed under the Creative Commons Attribution License, which permits unrestricted use, distribution, and reproduction in any medium, provided the original work is properly cited.

Mesh is a network topology that achieves high throughput and stable intercommunication. With great potential, it is expected to be the key architecture of future networks. Wireless sensor networks are an active research area with numerous workshops and conferences arranged each year. The overall performance of a WSN highly depends on the energy consumption of the network. This paper designs a new routing metric for wireless mesh sensor networks. Results from simulation experiments reveal that the new metric algorithm improves the energy balance of the whole network and extends the lifetime of wireless mesh sensor networks (WMSNs).

\section{Introduction}

Wireless sensor networks are one of the most rapidly evolving research and development fields for microelectronics. A wireless sensor network potentially comprises hundreds to thousands of nodes. These nodes are generally stationary after deployment, with the exception of a very small number of mobile sensor nodes, as shown in Figure 1. Wireless sensor networks characterize themselves in their distributed, dynamic, and self-organizing structure. Each node in the network can adapt itself based on environmental changes and physical conditions. Sensor nodes are expected to have low power consumption and simple structure characteristics, while possessing the ability of sensing, communicating, and computing. For conventional wireless networks, high degree of emphasis on mobility management and failure recovery is located in order to achieve high system performance. However, as the power of sensor nodes is usually supplied by battery with no continual maintenance and battery replenishment, to design a good protocol for WSNs, the first attribute that has to be considered is low energy consumption that could promise a long network lifetime. The recent advances of WSNs have made it feasible to realize low-cost embedded electric utility monitoring and diagnostic systems
$[1,2]$. In these systems, wireless multifunctional sensor nodes are installed on the critical equipment of the smart grid to monitor the parameters critical to each equipment's condition. Such information enables the smart-grid system to respond to varying conditions in a more proactively and timely manner. In this regard, WSNs play a vital role in creating a highly reliable and self-healing smart electric power grid that rapidly responds to online events with appropriate actions. The existing and potential applications of WSNs on smart grid span a wide range, including wireless automatic meter reading (WAMR), remote system monitoring, and equipment fault diagnostics.

There are lots of topology for wireless networks, such as star topology, mesh topology, and line topology. Among these topologies, mesh has large throughput and excellent stability, which is upcoming to become the model of the future network. Mesh networks, inspired from wireless neighborhood networks [3, 4], are composed of static wireless nodes that own several features, such as ample energy supplying, a distributed infrastructure, self-organizing and self-configuring capability, and ease and rapidity of network deployment, as shown in Figure 2. Each of these wireless nodes can be equipped with multiple radios, called a multiradio/multichannel node, and each of the radios can be 


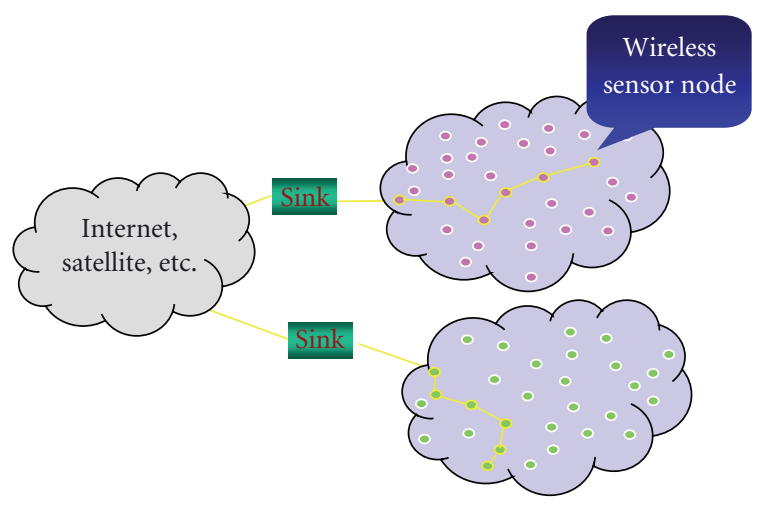

FIgURE 1: Wireless sensor network.

configured to a different channel to enhance network capacity. A wireless mesh network (WMN), possessing a more planned configuration, may be deployed to provide dynamic and cost-effective connectivity over a certain geographic area. Since its inception in the early years of this millennium, it has been in the limelight of all researchers.

The interest in wireless mesh networks has increased in recent years and several international standardization organizations are now developing specification for wireless mesh networking. IEEE 802.11 Task Group S (TGs), IEEE 802.16 Task Group, and IEEE 802.15.5th Task Group are standardizing wireless mesh networking. The Hybrid Wireless Mesh Protocol (HWMP) has been deemed as the mandatory routing protocol for WLAN mesh networks [5]. Although HWMP provides a mature routing scheme, merging the on-demand routing with the proactive routing, it does not present a method for WSNs, where limited energy is the crux and related works are barely done [6]. To achieve longevity in sensor networks, energy-aware architectures and protocols have been thoroughly investigated in the recent literature. The work in [7] introduced the NeLMUK algorithm to maximize the lifetime of 802.15.4based wireless sensor networks. The work in [8] improved the ZigBee mesh routing protocol for energy-efficiency usage and proposed a routing algorithm combining AODV with the node residual energy. Mesh topology is not discussed. Sereiko firstly proposed the concept of wireless mesh sensor network (WMSN) by deploying wireless routers to connect sensor networks [9].

This paper studies the method of searching for the optimum routing paths of wireless mesh sensor networks by modifying the routing metric in HWMP. A new routing metric is proposed, considering the route effect on the energy distribution of the network, called expected transmission energy (ETE). We simulate the new metric algorithm compared with HWMP and min-hop in NS3 to evaluate the performance of the proposed routing method. Through experiments, we confirm that our proposed algorithm has better performance in prolonging the lifetime of WMSNs.

The rest of this paper is organized as follows. In Section 2, we describe on-demand routing, proactive routing, and HWMP, pointing out their advantages and disadvantages.
Section 3 introduces the ETX and ETT metrics and presents the proposed ETE metric. In Section 4, we introduce the ETE into HWMP airtime metric and analyze the simulation results. Finally, Section 5 concludes this paper.

\section{Routing Protocols for Mesh Networks}

Routing protocols can be divided into two categories: ondemand routing and proactive routing. Different routing protocols have different costs in terms of message overhead and management complexity.

2.1. On-Demand Routing. Originally proposed for ad hoc networks, on-demand or reactive routing protocols (e.g., DSR [10], AODV [11], MCR [12], LBAR [13], and DLAR [14]) only create a route between a pair of source and destination nodes when the source node actually needs to send packets to the destination. Network-wide flooding is usually used to discover routes when they are needed. For ad hoc networks, since there are frequent link breaks caused by the mobility of nodes, flooding-based route discovery provides high network connectivity and relatively low message overhead compared with proactive routing protocols. In wireless mesh sensor networks, however, links usually have a much longer expected lifetime due to the static nature of nodes. Since the frequency of link breaks is much lower than the frequency of flow arrivals in mesh networks, flooding-based route discovery is both redundant and very expensive in terms of control message overhead. Therefore, pure on-demand routing protocols are generally not scalable and inappropriate for mesh networks.

2.2. Proactive Routing. In proactive routing protocols, each node maintains one or more tables containing routing information to every other node in the network. All nodes update these tables to maintain a consistent and up-to-date view of the network. When the network topology changes, the nodes propagate update messages throughout the network to maintain routing information about the whole network. These routing protocols differ in the methods by which packets are forwarded along routes. Every node maintains a routing table that indicates the next hops for the routes to all other nodes in the network. For a packet to reach its destination, it only needs to carry the destination address. Intermediate nodes forward the packet along its path based only on the destination address. Due to its simple forwarding scheme and low message overhead, proactive routing is dominant in wired networks.

2.3. Hybrid Wireless Mesh Protocol (HWMP). IEEE 802.11s deems HWMP as the mandatory routing protocol for WLAN mesh networks and optionally allows other routing protocols such as the Radio Aware Optimized Link State Routing (RA-OLSR) protocol. HWMP supports the two routing modes of on-demand and tree-based proactive to be cooperated. HWMP uses a common set of protocol primitives, generation, and processing rules inspired by the Ad hoc On-demand Distance Vector (AODV) protocol [11]. 


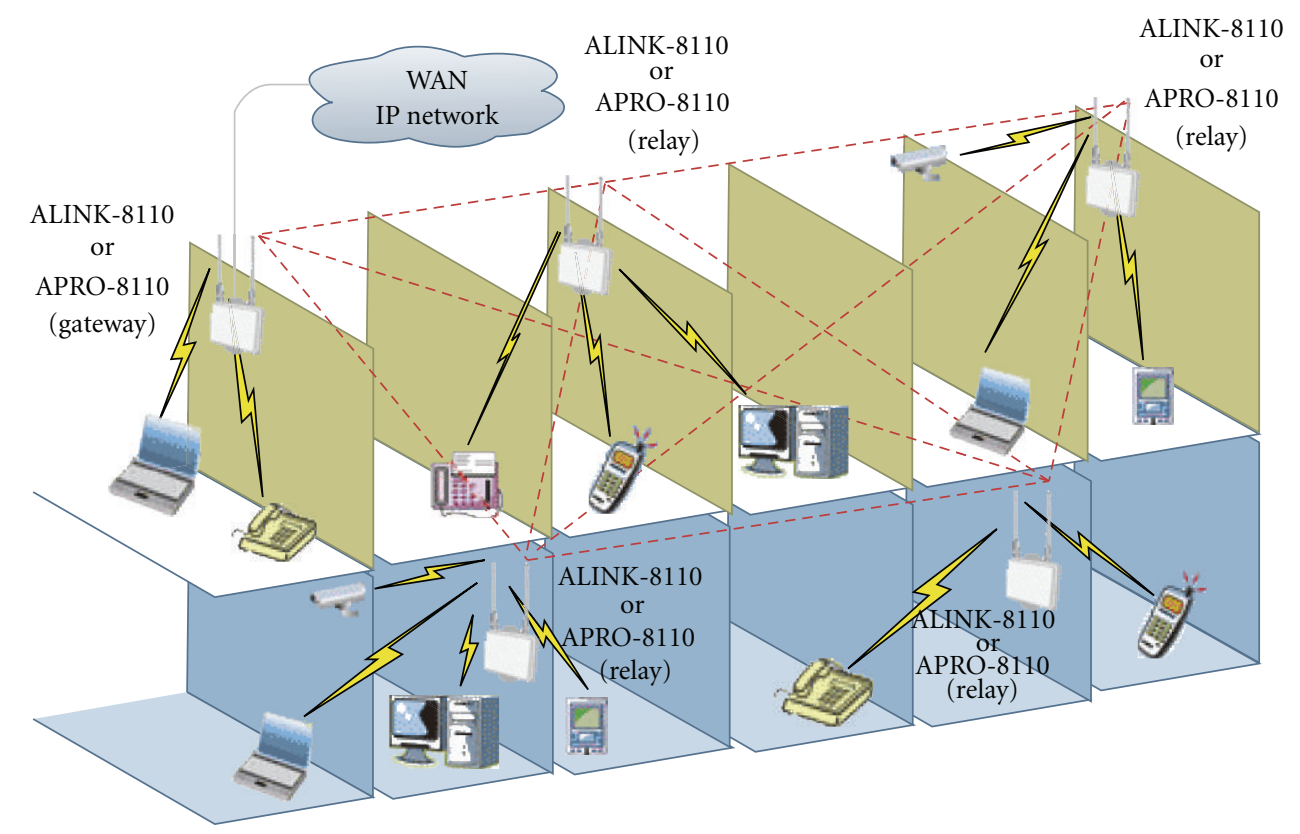

Figure 2: Wireless mesh network.

Four message frames are used in HWMP, namely, path request (PREQ), path reply (PREP), path error (PERR), and root announcement (RANN). Except for RANN that is only used in the proactive routing mode, these three message frames are mostly adopted from the AODV protocol.

HWMP, unlike traditional routing protocols on layer 3 with IP addresses, is operated on layer 2 using MAC addresses. When a routing protocol on layer 3 is used, data packets must be delivered to the IP layer to be routed. In the exchange between MAC addresses and IP addresses in the ARP table, overheads are also incurred. However, by adopting a layer 2 routing mechanism, HWMP can reduce overheads in forwarding data packets to destination nodes in WLAN mesh networks, based on multihop topologies. Consequently, high network throughputs can be achieved.

The operating method and the characteristics of the ondemand routing mode of HWMP are very similar to the existing AODV, except that HWMP uses layer 2 routing. In the on-demand routing mode, if a source node has no routing path to a destination node, it broadcasts a PREQ message inside the mesh network. The destination node that received the PREQ message sends a unicast PREP message back to the source node, and then a bidirectional routing path between the source and the destination nodes is established. During this procedure, the PREQ ID and the destination sequence number are used to prevent sending duplicated messages and to establish loop-free routing paths. The on-demand routing mode always provides the optimum routing paths by establishing its path when data transmission is required.

Figure 3 shows the instances of communication steps of mesh network. If mesh point 4 wants to communicate with mesh point 9 ,

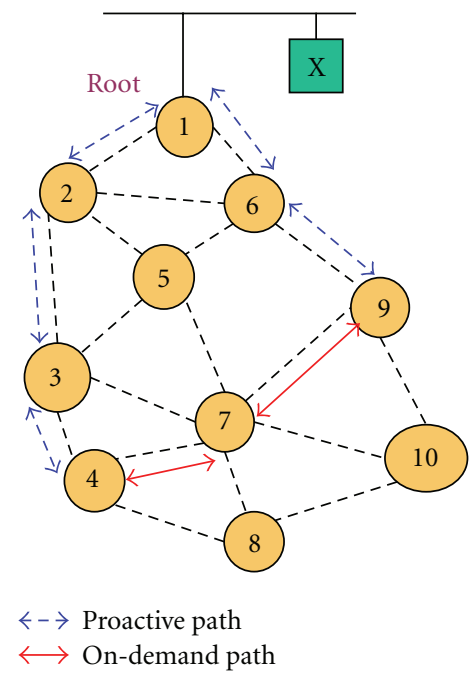

FIGURE 3: HWMP operation.

(1) MP 4 first checks its local forwarding table for an active forwarding entry to MP 9,

(2) if no active path exists, MP 4 may immediately forward the message on the proactive path toward the Root MP 1

(3) when MP 1 receives the message, it flags the message as "intra-mesh" and forwards on the proactive path to MP 9,

(4) when MP 9 receives the message, it may issue an on-demand RREQ to MP 4 to establish the best intramesh MP-to-MP path for future messages. 
If MP 4 wants to communicate with $\mathrm{X}$, who is outside of the current mesh cluster,

(1) MP 4 first checks its local forwarding table for an active forwarding entry to $\mathrm{X}$,

(2) if no active path exists, MP 4 may immediately forward the message on the proactive path toward the Root MP 1,

(3) when MP 1 receives the message, if it does not have an active forwarding entry to $\mathrm{X}$ it may assume the destination is outside the mesh and forward on other LAN segments according to locally implemented interworking.

No broadcast discovery is required when destination is outside of the mesh. This efficient routing meets the severe energy constrains of wireless sensor networks and the flexible connectivity of mobile nodes.

\section{Routing Metrics}

Router metrics can contain any number of values that help the router determine the best route among multiple routes to a destination. A router metric is typically based on information like path length, bandwidth, load, hop count, path cost, delay, Maximum Transmission Unit (MTU), reliability, and communications cost. In this section, we discuss 3 traditional routing metrics that have been proposed and HWMP routing metric in 802.11s. After that, we propose a new metric which counterbalances energy consumption of the network basing on HWMP metric.

\subsection{Traditional Routing Metric}

3.1.1. Hop Count. Hop count is the most commonly used routing metric in existing routing protocols such as DSR [10], AODV [11], DSDV, and GSR. It reflects the effects of path lengths on the performance of flows. Efficient algorithms can find loop-free paths with minimum hop count, since hop count metrics are isotonic. However, hop count does not consider the differences of the transmission rates and packet loss ratios in different wireless links, or the interference in the network. Hence, using a hop count metric may not result in a good performance.

3.1.2. Expected Transmission Count (ETX). ETX, proposed by De Couto et al. [15], is defined as the expected number of MAC layer transmissions that is needed for successfully delivering a packet through a wireless link. The weight of a path is defined as the summation of the ETX of all links along the path

$$
\mathrm{ETX}=\sum_{k-1}^{\infty} k p^{k-1}(1-p)=\frac{1}{1-p}
$$

Here, $k$ means that the transmission times for node $A$ send a packet to node $B$ successfully. And $p$ means error rate of the transmission.
Since both long paths and lossy paths have large weights under ETX, the ETX metric captures the effects of both packet loss ratios and path length. In addition, ETX is also an isotonic routing metric, which guarantees easy calculation of minimum weight paths and loop-free routing under all routing protocols. However, energy consumption of the devices is not taken into consideration in ETX.

3.1.3. Expected Transmission Time (ETT). The ETT routing metric, proposed by Draves et al. [16], improves ETX by considering the differences in link transmission rates. The ETT of a link $l$ is defined as the expected MAC layer duration for a successful transmission of a packet at link.

The weight of a path $p$ is simply the summation of the ETT's of the links on the path. The relationship between the ETT of a link $l$ and ETX can be expressed as

$$
\mathrm{ETT}_{l}=\operatorname{ETX}_{l} \frac{s}{b_{l}}
$$

where $b_{l}$ is the transmission rate of link $l$ and $s$ is the packet size. Essentially, by introducing $b_{l}$ into the weight of a path, the ETT metric captures the impact of link capacity on the performance of the path. Similar to ETX, ETT is also isotonic. However, the remaining drawback of ETT is that it still does not fully capture energy consumption in the network. For example, ETT may choose a path in which energy of the devices is quite low. Though it may achieve high throughput, the lifetime of the WMSN may be awfully short as energy consumption focuses on some nodes.

3.2. HWMP Airtime Metric. In HWMP, the cost function for establishment of the radio-aware paths is based on airtime cost. Airtime cost reflects the amount of channel resources consumed by transmitting the frame over a particular link. This measure is approximate and designed for ease of implementation and interoperability.

The airtime cost for each link is calculated as

$$
c_{a}=\left[O_{c a}+O_{p}+\frac{B_{t}}{r}\right] \frac{1}{1-e_{p t}},
$$

where $O_{c a}, O_{p}$, and $B_{t}$ are constants listed in Table 1, and the input parameters $r$ and $e_{p t}$ are the bit rate in $\mathrm{Mb} / \mathrm{s}$ and the frame error rate for the test frame size $B_{t}$, respectively. The rate $r$ represents the rate at which the mesh point would transmit a frame of standard size $\left(B_{t}\right)$ based on current conditions, and its estimation is dependent on local implementation of rate adaptation; the frame error rate $e_{p t}$ is the probability that when a frame of standard size $\left(B_{t}\right)$ is transmitted at the current transmission bit rate $(r)$, the frame is corrupted due to transmission error, and its estimation is a local implementation choice. Frame drops due to exceeding TTL should not be included in this estimate as they are not correlated with link performance. This metric algorithm only takes the communication channel between nodes into account. However, it does not consider the condition of node's itself. 
TABLE 1: Airtime cost constants.

\begin{tabular}{|c|c|c|c|}
\hline Parameter & $\begin{array}{c}\text { Value } \\
(802.11 \mathrm{a})\end{array}$ & $\begin{array}{c}\text { Value } \\
(802.11 \mathrm{~b})\end{array}$ & Description \\
\hline$O_{c a}$ & $75 \mathrm{~ms}$ & $335 \mathrm{~ms}$ & $\begin{array}{l}\text { Channel access } \\
\text { overhead }\end{array}$ \\
\hline$O_{p}$ & $110 \mathrm{~ms}$ & $364 \mathrm{~ms}$ & Protocol overhead \\
\hline$B_{t}$ & 8224 & 8224 & $\begin{array}{l}\text { Number of bits in } \\
\text { test frame }\end{array}$ \\
\hline$r$ & & & $\begin{array}{l}\text { Current bit rate in } \\
\text { use }\end{array}$ \\
\hline$e_{p t}$ & & & $\begin{array}{l}\text { Packet error rate at } \\
\text { the current bit rate }\end{array}$ \\
\hline
\end{tabular}

3.3. Expected Transmission Energy (ETE). All the algorithms mentioned above do not take the energy factor into account which is one of the most important problems of wireless sensor network. Our assumption is that all nodes are considered equally important in a WMSN. If a node died, we will lose control of a certain space in the sensing field. To prevent this situation, we would like to ensure that no node consumes energy at a rate significantly higher than other nodes, while simultaneously keeping the average power consumption rate low.

This conception can be formulated as two important parameters:

$$
\begin{gathered}
\sigma_{E}^{2}=\frac{\sum_{i=1}^{n}\left(E_{i}-\bar{E}\right)}{n}, \\
\bar{E}_{c}=\frac{\sum_{i=1}^{n_{j}} E_{i c}}{n_{j}} .
\end{gathered}
$$

$E_{i}$ is the remaining energy of node $i$ after the transmission, $E_{i c}$ is the energy consumption of the node $i$ in the transmission which related to the distant, $n$ is the number of nodes of the whole network, and $n_{j}$ is the number of nodes along the selected route.

The route judgment can be concluded as the following four steps.

(1) $\bar{E}_{c}$ must be less than some maximum budget threshold $\bar{E}_{t}$.

(2) $\sigma_{E}^{2}$ should be minimized after the transmission.

(3) Once (1) and (2) are satisfied, the less $\sum_{i=1}^{n_{j}} E_{i c}$ it has, the better route it is.

(4) Once a node's energy is below a designated threshold, the node will not join the calculation of route metric which means it only sends his new packets and will not forward packets for others.

The routing metrics must ensure that optimum paths can be found by efficient algorithms with polynomial complexity. However, the expression of the $\sigma_{E}^{2}$ makes the algorithm too complex to realize. So the algorithm needs to be simplified. The minimization of $\sigma_{E}^{2}$ is equal to choosing route with the maximum $\bar{E}_{c}$. If the number is different, the situation could be simplified as Figure 4 . The path with powerful nodes in

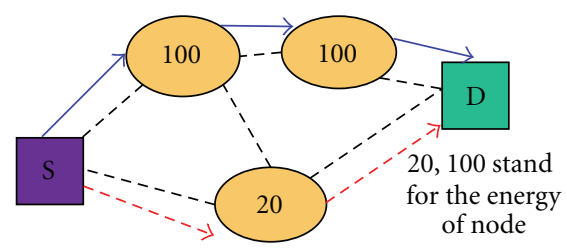

FIgURE 4: Route selection based on ETE metric.

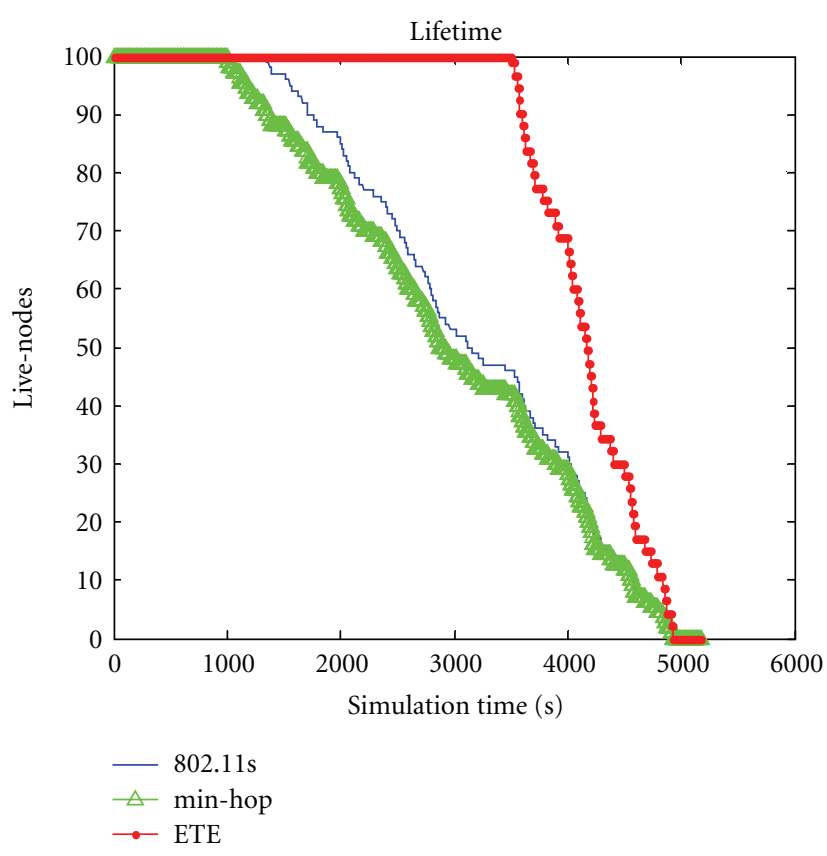

FIGURE 5: Lifetime of WMSN.

solid line will be chosen as the transmission path. While the dotted line path, though has less hop count, does not help to do energy balance of the network and will not be chosen.

Focusing the energy consumption on the route consisting of nodes with more power will help to the balance of the energy distribution of the whole network. But it will take a further path which contains more powerful nodes that help to increase the average energy $\bar{E}_{c}$. So, our simplified metric can be modified as $\sum_{i=1}^{n_{j}} 1 / E_{i}$. To combine with the $802.11 \mathrm{~s}$ airtime metric, it is normalized as $\sum_{i=1}^{n_{j}} 1 /\left(100\left(E_{i} / E_{\text {init }}\right)\right)$ and $\bar{E}_{c}$ is normalized as $\sum_{i=1}^{n_{j}}\left(E_{i c} / E_{\text {init }}\right) / n_{j}$. And the full expression of our metric at last could be concluded as the following:

$$
c_{a}^{\prime}=\left[O_{c a}+O_{p}+\frac{B_{t}}{r}+\sum_{i=1}^{n_{j}} \frac{E_{\mathrm{init}}}{100 E_{i}}\right] \frac{1}{1-e_{p t}}+\frac{\sum_{i=1}^{n_{j}}\left(E_{i c} / E_{\mathrm{init}}\right)}{n_{j}} .
$$

As $1 /\left(1-e_{p t}\right)<1, \sum_{i=1}^{n_{j}}\left(E_{i c} / E_{\text {init }}\right) / n_{j}$ contributes less than $\sum_{i=1}^{n_{j}}\left(E_{\text {init }} / 100 E_{i}\right)$ to the result of $c_{a}^{\prime}$.

In case there exist two routes with approximately equal energy. To prevent extra energy consumption in route establishment, the route metric will not be recalculated until the tenth transmission on the old route. 


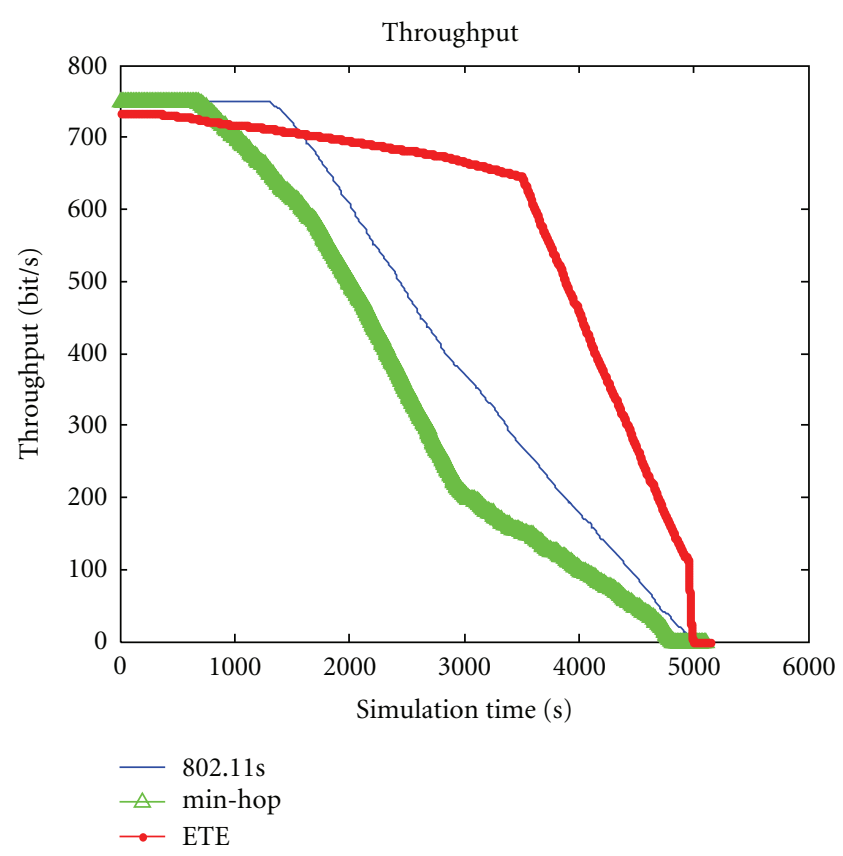

Figure 6: Throughput of WMSN.

\section{Simulation and Analysis}

All of our simulations are performed in the NS3 simulator. And the figures are printed by Matlab after the process of awk script. One hundred nodes are regularly placed over a $10 \times 10$ grid. The sources and destinations of the data flows are randomly located in the mesh network while the root of mesh network is designated. To simplify the simulation, we set the initial energy of a node 100. If a node sends a packet, it will cost 1 , same as its receiving process. This means the node will lose 2 of energy when it forwards a packet. If a node's energy is less than $20 \%$ of $E_{\text {init }}$, this node will refuse to forward packets for other nodes. The remaining energy of it will only be used to send its own new data.

4.1. Lifetime. In Figure 5, the line with triangle, the leftmost one, represents the min-hop metric, and its lifetime is the shortest. This is because it just considers the minimization of hop count. However, even though some paths have less hop counts, the error rate is awful. Thus, forcing the nodes to retransmit their packets and causing a waste of energy. The line with asterisk in the rightmost represents the improved algorithm. Simulation result shows that the modified algorithm balances the energy consumption more effectively, that is, the lifetime of the WMSN is prolonged.

4.2. Throughput. As is shown in Figure 6, ETE achieves higher throughput. In the improved algorithm, the nodes with energy under the threshold will refuse to forward packets for others, helping extend the lifetime of low power nodes and balance the energy distribution of the network. Moreover, these nodes with low power will consume energy only when they have their own new data sent. Hence, we have a higher throughput in the last part of the simulation, the rightmost one. Compared with ETE, low power nodes in 802.11 s and min-hop routing die away more quickly and the total amount of data the nodes sensed become less which pull down the throughput.

\section{Conclusion}

In this paper, we study the traditional route metrics and point out their common deficiency-neglecting energy consumption. We proposed a new route metric called ETE and introduced it into 802.11 s airtime metric. The simulation reveals that the new algorithm improves the energy balance of the whole network and extends the lifetime of wireless mesh sensor network. In the future, the proposed algorithm may be further considered for multichannel wireless mesh sensor networks. In addition, it will be used in the existing and potential applications of WMSNs on smart grid.

\section{Acknowledgments}

The authors would like to thank the reviewers for their detailed comments on earlier versions of this paper. This work was supported by Shanghai Leading Academic Discipline project (S30108, 08DZ2231100), Shanghai Education Committee (09YZ33), Shanghai Science Committee $(08220510900,10511501303)$ and Shanghai SMIT Fund.

\section{References}

[1] L. Lo Bello, O. Mirabella, and A. Raucea, "Design and implementation of an educational testbed for experiencing with industrial communication networks," IEEE Transactions on Industrial Electronics, vol. 54, no. 6, pp. 3122-3133, 2007.

[2] B. Lu and V. C. Gungor, "Online and remote motor energy monitoring and fault diagnostics using wireless sensor networks," IEEE Transactions on Industrial Electronics, vol. 56, no. 11, pp. 4651-4659, 2009.

[3] R. Karrer, A. Sabharwal, and E. Knightly, "Enabling large-scale wireless broadband: the case for TAPs," in Proceedings of the Workshop on Hot Topics in Networks (HotNets '03), no. 1, pp. 27-32, Cambridge, Mass, USA, 2003.

[4] V. Gambiroza, B. Sadeghi, and E. W. Knightly, "End-toend performance and fairness in multihop wireless backhaul networks," in Proceedings of the 10th Annual International Conference on Mobile Computing and Networking (MobiCom '04), pp. 287-301, October 2004.

[5] IEEE Std 802.11TM-2007, "Wireless LAN Medium Access Control (MAC) and Physical Layer (PHY) Specifications," IEEE Computer Society, June 2007.

[6] J. D. Camp and E. W. Knightly, "The IEEE 802.11s extended service set mesh networking standard," IEEE Communications Magazine, vol. 46, no. 8, pp. 120-126, 2008.

[7] M. U. Ilyas and H. Radha, "Increasing network lifetime of an IEEE 802.15.4 wireless sensor network by energy efficient routing," in Proceedings of the IEEE International Conference on Communications (ICC '06), pp. 3978-3983, July 2006.

[8] F. Zhang, H. Zhou, and X. Zhou, "A routing algorithm for zigbee network based on dynamic energy consumption decisive path," in Proceedings of the International Conference 
on Computational Intelligence and Natural Computing (CINC '09), pp. 429-432, June 2009.

[9] P. Sereiko, "Wireless Mesh Sensor Networks Enable Building Owners, Managers, and Contractors to Easily Monitor HVAC Performance Issues," 2004, http://www.automatedbuildings. com/news/jun04/articles/sensicast/Sereiko.htm.

[10] D. B. Johnson and D. A. Maltz, "Dynamic source routing in AdHoc wireless networks," in Mobile Computing, vol. 353, Kluwer Academic, Boston, Mass, USA, 1996.

[11] C. Perkins, "Ad-Hoc on-demand distance vector routing," in Proceedings of the IEEE Military Communications Conference on Ad Hoc Networks (Milcom '97), 1997.

[12] P. Kyasanur and N. Vaidya, "Multi-channel wireless networks: capacity and protocols," Tech. Rep., University of Illinois at Urbana-Champaign, Urbana, Ill, USA, 2005.

[13] H. Hassanein and A. Zhou, "Routing with load balancing in wireless ad hoc networks," in Proceedings of the 4th ACM International Workshop on Modeling, Analysis and Simulation of Wireless and Mobile Systems (ACM MSWiM '01), pp. 89-96, July 2001.

[14] C. Perkins, E. Belding-Royer, and S. Das, "Ad Hoc OnDemand Distance Vector (AODV) Routing," IETF RFC 3561, July 2003.

[15] D. S. J. De Couto, D. Aguayo, J. Bicket, and R. Morris, "A HighThroughput Path Metric for Multi-Hop Wireless Routing," in Proceedings of the Ninth Annual International Conference on Mobile Computing and Networking (MobiCom '03), pp. 134146, September 2003.

[16] R. Draves, J. Padhye, and B. Zill, "Routing in multi-radio, multi-hop wireless mesh networks," in Proceedings of the 10th Annual International Conference on Mobile Computing and Networking (MobiCom '04), pp. 114-128, October 2004. 

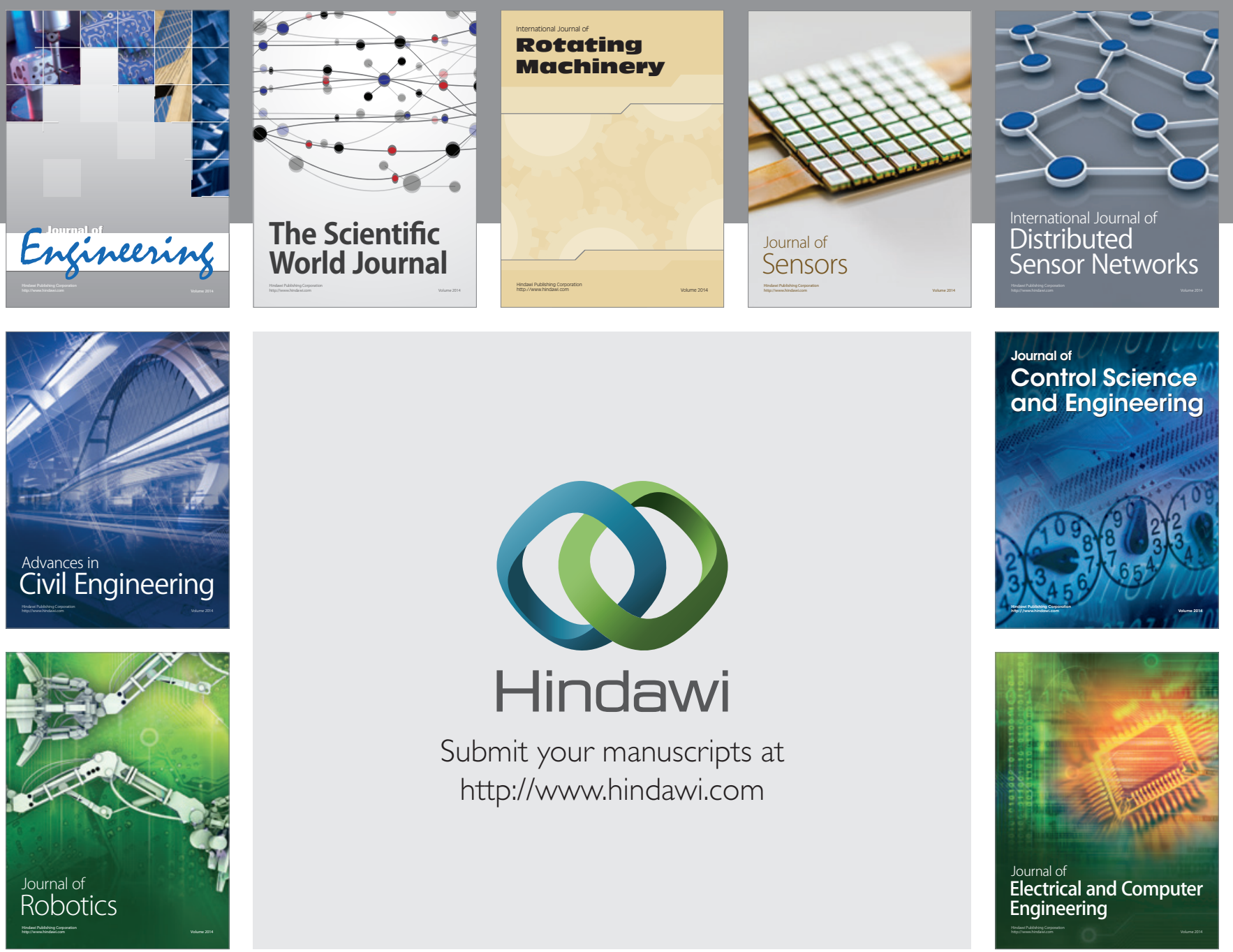

Submit your manuscripts at

http://www.hindawi.com
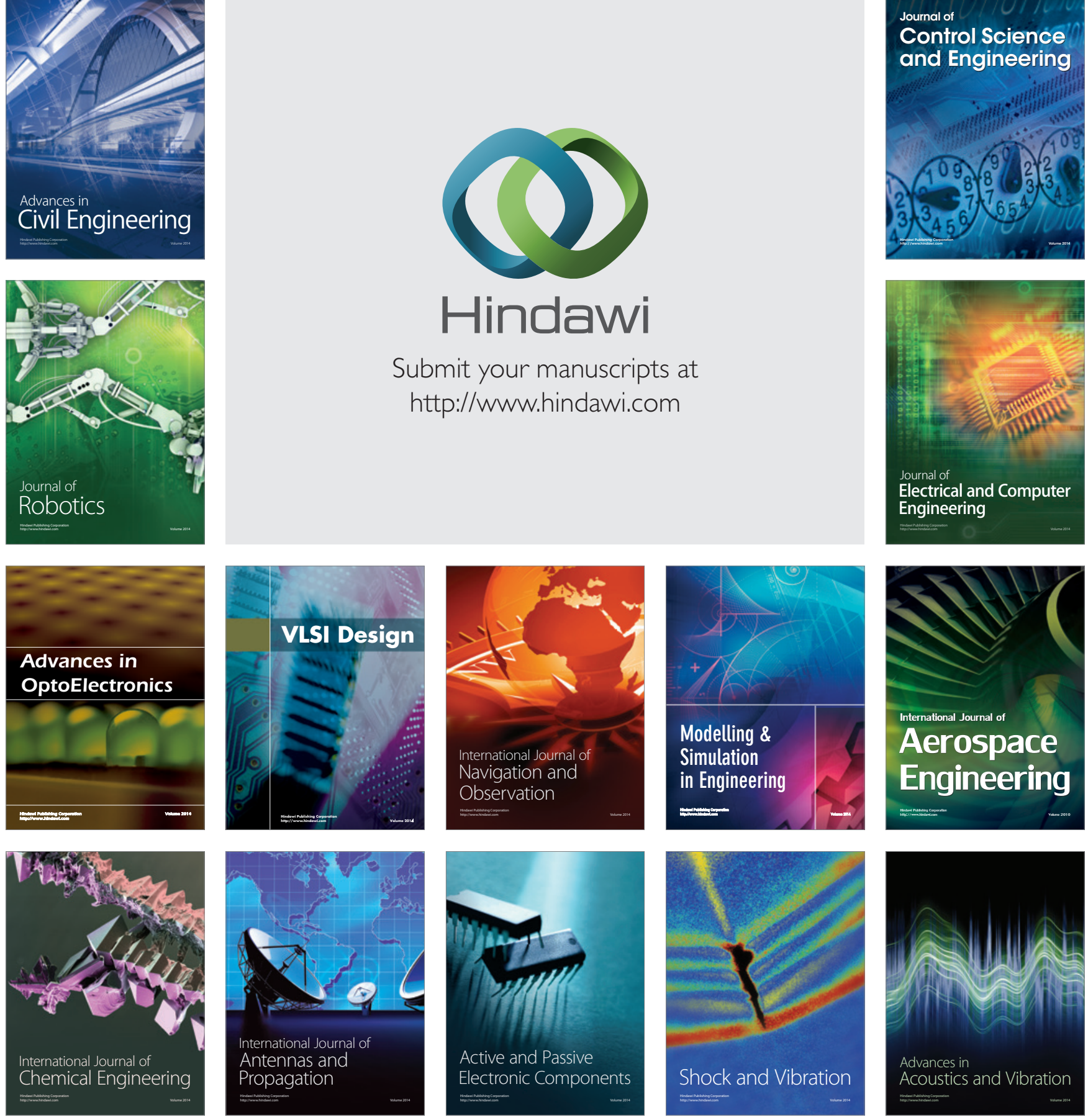